\title{
Array-CGH fine mapping of minor and cryptic HR-CGH detected genomic imbalances in 80 out of 590 patients with abnormal development
}

\author{
Helle Lybæk ${ }^{*, 1}$, Leonardo A Meza-Zepeda ${ }^{2}$, Stine H Kresse ${ }^{2}$, Trude Høysæter ${ }^{1}$, \\ Vidar M Steen $^{1,3}$ and Gunnar Houge ${ }^{1}$
}

${ }^{1}$ Center for Medical Genetics and Molecular Medicine, Haukeland University Hospital, Helse Bergen HF, Norway;
${ }^{2}$ Department of Tumor Biology, Rikshospitalet-Radiumhospitalet Medical Center, Oslo, Norway; ${ }^{3}$ Department of
Clinical Medicine, University of Bergen, Bergen, Norway

During a 6-year period, 590 patients suspected of having a minor or cryptic genomic imbalance as the cause of mental retardation with dysmorphic signs $+/-$ malformations have been investigated with highresolution comparative genomic hybridisation (HR-CGH) in our diagnostic laboratory. Thirty-six patients had a small chromosomal aberration detected by routine karyotyping, and 554 patients had a normal G-banded karyotype. In the latter group, a genomic imbalance was detected by HR-CGH in 40 patients (7.2\%): 29 deletions, 3 duplications, 4 unbalanced translocations, and 4 occult trisomy mosaicisms. When microarray-based comparative genomic hybridisation (array-CGH) became available, all HR-CGH-positive samples were also investigated by $1 \mathrm{Mb}$ resolution array-CGH for more precise mapping. From the 514 patients with normal HR-CGH findings, a subset of 20 patients with particularly high suspicion of having a chromosomal imbalance was selected for array-CGH. In four of them $(20 \%)$, an imbalance was detected: three deletions and one duplication. Of note, 73 out of the 80 array-CGH mapped patients had a de novo chromosomal rearrangement $(91 \%)$. Taken together, this work provides phenotype-genotype information on 80 patients with minor and cryptic chromosomal imbalances.

European Journal of Human Genetics (2008) 16, 1318-1328; doi:10.1038/ejhg.2008.78; published online 7 May 2008

Keywords: $\mathrm{CGH}$; array- $\mathrm{CGH}$; mental retardation; microdeletion; microduplication

\section{Introduction}

In Europe, mental retardation with or without dysmorphic signs and malformations is recognised in around $1 \%$ of children. ${ }^{1-3}$ The true incidence may be higher due to nonascertainment. An unbalanced karyotype can be found in $10 \%{ }^{4}$ to $16 \%^{5}$ of these individuals by routine chromosome analysis (microscopy of G-banded metaphases from a T-lymphocyte blood culture). Other patients have a cryptic (ie invisible) chromosomal imbalance detectable by

*Correspondence: $\mathbf{H}$ Lybæk, Center for Medical Genetics and Molecular Medicine, Haukeland University Hospital, N-5021 Bergen, Norway. Tel: +4755 9770 30/ + 47559754 75; Fax: + 47559754 79; E-mail: helle.lybak@helse-bergen.no

Received 13 December 2007; revised 11 March 2008; accepted 13 March 2008; published online 7 May 2008 chromosome-based high-resolution comparative genomic hybridisation (HR-CGH). ${ }^{6,7}$ We and others have found that deletions above $\sim 3 \mathrm{Mb}$ can be detected by HR-CGH. ${ }^{8}$ Duplications are usually more difficult to detect. In recent years, CGH profiles have been determined using genomic microarrays instead of metaphase chromosomes to obtain higher sensitivity. ${ }^{9-14}$ The resolution is no longer limited by the quality of the normal metaphase spreads, but by the density of genomic clones. Many laboratories have employed bacterial artificial chromosome (BAC)-based arrays with an average clone distance of $1 \mathrm{Mb}$ for screening patients with developmental delay and/or patients suspected for having a cryptic chromosomal imbalance, hereafter called $1 \mathrm{Mb}$ array-CGH. The $1 \mathrm{Mb}$ array-CGH pick-up rate for clinically relevant chromosomal 
imbalances in mentally retarded patients with normal karyotypes was $8.4 \%$ when data from five different studies with a total of 332 patients were taken into account. ${ }^{10}$ Using a more limited 831-clone BAC array targeted to regions of known clinical relevance, a genomic alteration was found in $5.6 \%$ of 1500 consecutive cases with a variety of developmental problems. ${ }^{15}$

In our laboratory, we have used HR-CGH as a screening method for cryptic chromosomal imbalances for several years. In an initial study, we found a cryptic chromosomal imbalance in 5 out of 50 patients with abnormal development and normal karyotypes, ${ }^{7}$ in line with other observations. ${ }^{6}$ As an extension of this study and to map minor imbalances more accurately, we have recently used $1 \mathrm{Mb}$ array-CGH to investigate all HR-CGH findings obtained during the past 6 years. Thus, this work gives detailed information on the position of the genomic imbalances in 80 patients with abnormal phenotypes, adding data on phenotype-genotype correlations.

\section{Patients and methods Patients}

Blood samples from 590 patients (age range 1 - 58 years), all except 3 being mentally retarded, and most also having dysmorphic features and/or malformations, were received for analysis at the Center for Medical Genetics and Molecular Medicine, Haukeland University Hospital, during a 6-year period (2001-2006). Blood samples were referred to us from the whole of Norway by medical geneticists or experienced paediatricians. After conventional G-banded karyotyping, the samples were examined by HR-CGH analysis. This was performed either because the observed chromosomal abnormality was small and doubtful or difficult to classify, or because the patient phenotype was suggestive of a chromosomal abnormality despite the karyotype being normal. When array-CGH became available, all HR-CGH-positive samples were investigated by $1 \mathrm{Mb}$ array-CGH for fine mapping. In addition, $1 \mathrm{Mb}$ array-CGH analysis was carried out on a subgroup of 20 patients with strong clinical impression of having a genomic imbalance despite normal findings on G-banding and HR-CGH. The layout of the study is summarised in Figure 1. Parental analyses were carried out to investigate whether the finding was de novo or the result of a balanced rearrangement in one of the parents. Parental DNA was not available for case 32 (Table 1). Many patients were later referred to us for clinical evaluation and genetic counselling. In other patients, more detailed clinical information was asked for. The patients' phenotypes are summarised in Tables $1-3$.

\section{HR-CGH}

Chromosome-based HR-CGH was performed as described previously. ${ }^{7}$ Briefly, patient DNA and normal reference
DNA (800 ng each) were labelled with fluorescein isothiocyanate-12-dUTP and Texas Red-5-dUTP (NEN Life Science Products Inc., USA), respectively. Commercial reference DNA was used, consisting of a pool from either 10 normal female donors or 10 normal male donors (Promega $\mathrm{GmbH}$, Germany). The labelled DNAs together with $20 \mu \mathrm{g}$ of human Cot-1 DNA (Invitrogen Inc., USA) were hybridised onto normal metaphase chromosomes (from the same individual). Sex-matched hybridisations were performed. Analysis was performed using the high-resolution CGH analysis software that is part of the CytoVision System (Applied Imaging, UK). Imbalances were scored as a deviation from the standard reference interval CGH ratio profiles at $99.5 \%$ confidence intervals. ${ }^{16}$ The CGH results were verified either by re-examination of G-banded highquality chromosomes (band level above 600) or by BACbased fluorescence in situ hybridisation (FISH) analysis. ${ }^{7}$

\section{Array-CGH}

For array-CGH, a BAC/PAC clone set consisting of approximately 3500 clones with an average interclonal distance of $1 \mathrm{Mb}$ was obtained from the Wellcome Trust Sanger Institute (UK). ${ }^{17}$ Production of genomic $1 \mathrm{Mb}$ arrays based on the clone set was done by the Norwegian Microarray Consortium (NMC; www.microarray.no) at the RR-HF/UiO Microarray Core Facility in Oslo, Norway. Arrays were produced as described previously, ${ }^{18}$ but tile path resolution on chromosome 1q was not included in our arrays. Each clone is represented by four DNA spots on the array. ArrayCGH was performed as described previously. ${ }^{18}$ Briefly, patient DNA and normal reference DNA (500 ng each) were labelled with Cy3-dCTP and Cy5-dCTP (NEN Life Science Products), respectively. Commercial reference DNA described above was used (Promega). The labelled DNAs together with $135 \mu \mathrm{g}$ of human Cot-1 DNA (Invitrogen) were hybridised onto the array. Sex-matched hybridisations were carried out. Arrays were scanned in an Agilent G2565B scanner (Agilent Technologies, USA), and images were analysed using GenePix Pro 5.0 software (Molecular Devices Corp., USA). Further data processing, including normalisation and filtering, was performed using the M-CGH software. ${ }^{19}$ In brief, spots with intensities lower than the background, or signal intensities below local background plus twice the standard deviation (SD) of the background, or replicates with $\mathrm{SD}>0.2$, were excluded from the analysis. Classification (gains, normals, and losses) and calculation of the $\log 2$ ratio thresholds for the amplicons were computed by the M-CGH software. ${ }^{19}$ BAC clone information was based on the Ensembl database v44 (www.ensembl.org), NCBI build 36.2.

Determination of the size of a deletion or duplication The $1 \mathrm{Mb}$ distance between the BAC clones is an average. The real distance varies depending on chromosomal location, and a breakpoint may also be in a region partly 


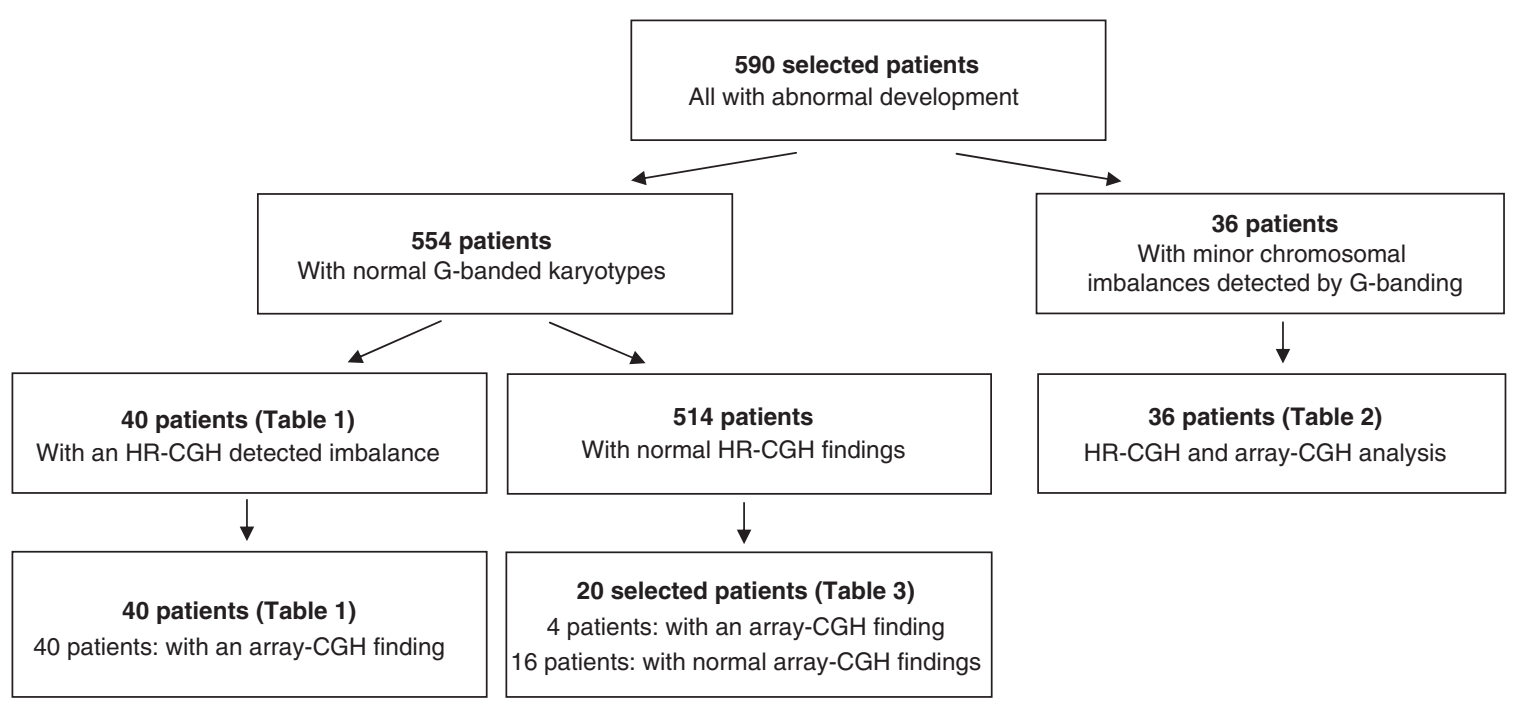

Figure 1 Study layout.

covered by an abnormal clone. Therefore, the sizes of the deletions and duplications (Tables $1-3$ ) are given both as the minimum and maximum sizes. The terminal clones of the imbalance define the minimum size and the position of the imbalance, and the normal flanking clones define the maximum size. The end coordinates of the clones were used for size determination. Aberration calls were based on two or more consecutive clones.

\section{Results}

In our sample of 590 patients with abnormal development, which has been screened for minor or cryptic chromosomal imbalances by G-banding and HR-CGH, we applied array$\mathrm{CGH}$ on a subset of 96 patients, using $1 \mathrm{Mb}$ resolution BAC clone arrays. The patients' phenotypes are summarised in Tables $1-3$. The specificity of the $1 \mathrm{Mb}$ BAC arrays was tested in advance by running a total of 10 normal-versus-normal hybridisations with DNA from normal male or female blood donors (data not shown). A low level of background noise, that is, clones variably showing a signal intensity ratio outside the limits defined for normal DNA copy numbers, was detected. On average, 15 non-consecutive single clones (0.4\%), behaving differently from experiment to experiment in the normal-versus-normal hybridisations, were detected. To test the sensitivity and reproducibility of the array slides, DNA samples from two patients with deletions of known size after FISH mapping (case 6 in Table 1 and case 69 in Table 2) were run for analysis four times each. The genomic ratio profiles of the four repeated experiments in both cases were consistent (data not shown).

\section{Detection of cryptic chromosomal imbalances and} occult trisomy mosaicisms

Among the 590 patients described above, 36 had a small visible chromosome aberration detected by G-banding and
554 patients had a normal karyotype. When genomic BAC arrays became available from the NMC, $1 \mathrm{Mb}$ array-CGH analysis was used for fine mapping of all HR-CGH findings and for examination of selected patients with normal findings on both G-banding and HR-CGH. In the group of 554 patients having a normal karyotype, HR-CGH analysis detected a genomic imbalance in 40 of them (7.2\%; Table 1). Of note, four patients had occult mosaicism: three for trisomy 9 and one for trisomy 14 . The percentage of mosaicism in these patients (cases 25, 26, 27 and 33; Table 1) was determined by interphase FISH to be $21,23,15$ and $36 \%$, respectively.

Among the 40 patients, all imbalances were known to be de novo aberrations except cases 5, 11 and 20 (parent translocation carrier) and case 32 (unknown). Of the 40 patients, $72 \%(29 / 40)$ had deletions, 10\% (4/40) had occult trisomy mosaicisms, $10 \%$ (4/40) had cryptic translocations and $8 \%(3 / 40)$ had duplications (Table 1). The mean minimum size of an HR-CGH-detected isolated deletion was $6.0 \mathrm{Mb}$ (Table 1), whereas the mean maximum size was $8.5 \mathrm{Mb}$. For the three isolated duplications found (Table 1), the mean minimum size was $5.6 \mathrm{Mb}$ and the mean maximum size was $8.9 \mathrm{Mb}$. The theoretical size maximum of the smallest duplication detected (case 12 in Table 1) was $5.3 \mathrm{Mb}$.

In the group of 36 patients with small chromosome aberrations detected by G-banding, HR-CGH and arrayCGH were applied for better characterisation (Table 2). Among these patients, all imbalances were known to be de novo aberrations except cases 62 (parent translocation carrier) and 69 (familial inversion). Of the 36 patients, 13 had deletions, 12 had duplications and 11 had translocations/complex single-chromosome rearrangements. Thus, in the group of 40 patients with normal G-banded karyotypes (Table 1), a deletion (29/40) was a 10 times 
Table 1 Array-CGH fine mapping of chromosomal imbalances detected by HR-CGH, but not by routine G-banding

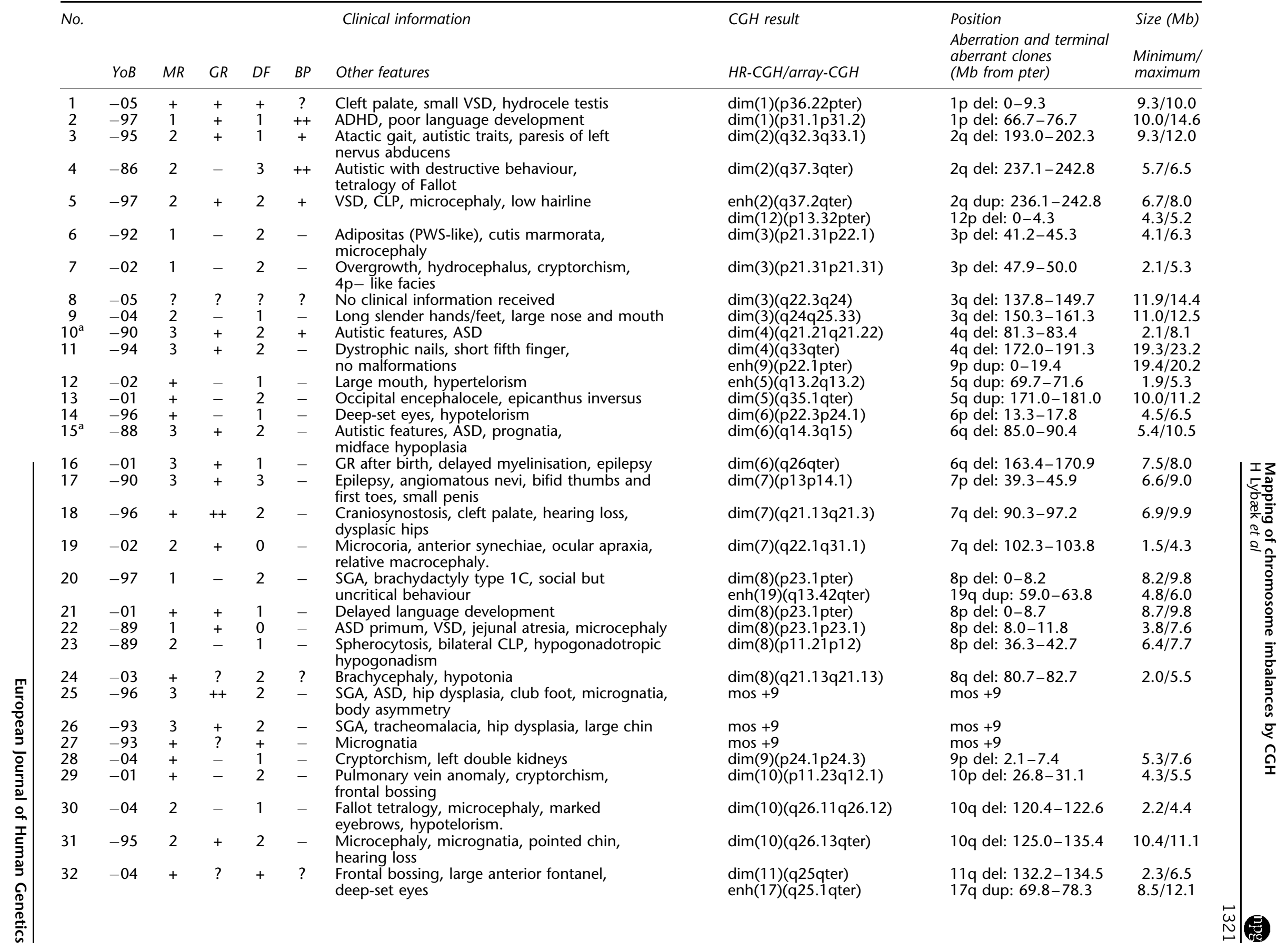




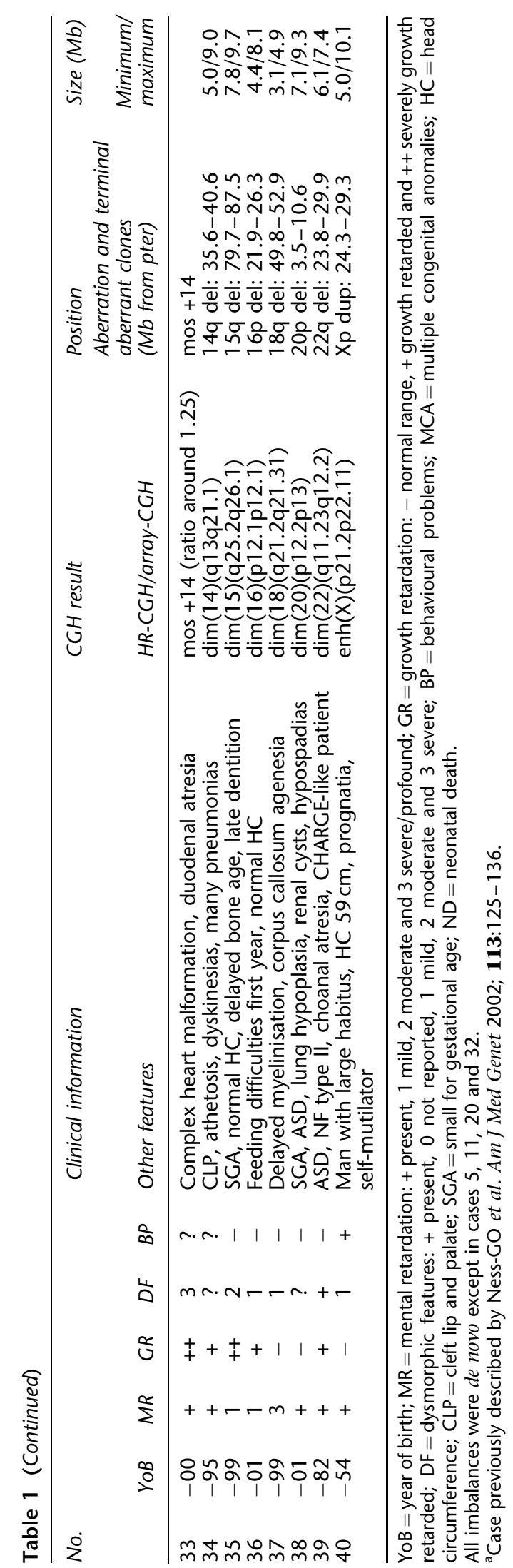

more common finding than duplication (3/40), whereas in the group of 36 patients with aberrations detectable by Gbanding (Table 2), small deletions and duplications were equally common findings. In Table 1 , all six non-mosaic patients known to have severe mental retardation had deletions (mean minimum size $7.3 \mathrm{Mb}$ ). In contrast, in Table 2, all six patients known to have severe mental retardation had duplications (mean minimum size $=$ 15.7 Mb).

In Figure 2, the location and size of all deletions and duplications are shown apart from the four cases with occult trisomy mosaicism. A total of $91 \%$ of the imbalances were de novo. Except for two patients (case 69; Table 2 and case 80; Table 3), all single-chromosome imbalances were de novo (Figure 2). Imbalances were found on all chromosomes. The size and number of imbalances varied greatly between chromosomes, and the majority of the imbalances were found on chromosomes 2-10, 13 and 14. When only the interstitial chromosome rearrangements were counted, the imbalances were more equally spread on all chromosomes.

As expected, the array-CGH results showed that the plots made by the HR-CGH software could be misleading. In 3 of the 40 patients with cryptic imbalances (Table 1), HR-CGH indicated the position of a deletion or duplication that missed its true position: The $5 \mathrm{q} 13$ duplication in case 12 , the subterminal $9 p$ deletion in case 28 , and the $10 q 26$ deletion in case 30 . In case 58 (Table 2), the HR-CGH finding was both incorrect and incomplete. Here, a terminal deletion combined with a subterminal duplication on 7q was only shown as a subterminal 7q duplication by the HR-CGH software. The finding of a terminal deletion combined with a subterminal duplication suggests that the mechanism might have been a breakage-fusionbridge cycle after $7 \mathrm{q}$ arm fusion. ${ }^{20}$ In two patients with small but visible imbalances (Table 2), the HR-CGH software excluded the subtelomeric regions from the imbalance, $^{16}$ for example, case 72 , where a terminal $19 q$ duplication that appeared to be interstitial (Figure 3a) and, case 74, where the HR-CGH software suppressed the terminal part of a $22 \mathrm{q} 13$ deletion. This demonstrates that HR-CGH software may overlook even visible subtelomeric imbalances.

\section{Array-CGH on a subset of patients with normal karyotype and HR-CGH}

Twenty patients with a phenotype strongly suggesting a chromosomal abnormality despite normal findings on both G-banding and HR-CGH were selected for $1 \mathrm{Mb}$ array-CGH. In four of them (20\%), a chromosomal imbalance was found: three deletions (mean minimum size $3.2 \mathrm{Mb}$, range $3.1-3.3 \mathrm{Mb}$ ) and one duplication (minimum size $8.7 \mathrm{Mb}$ ) (Table 3 ). All findings were verified by array-CGH analysis on CytoChip-arrays (BlueGnome, UK), a commercial BAC array with $1 \mathrm{Mb}$ resolution (data not 
Table 2 Array-CGH fine-mapping of chromosomal aberrations detected by routine G-banding

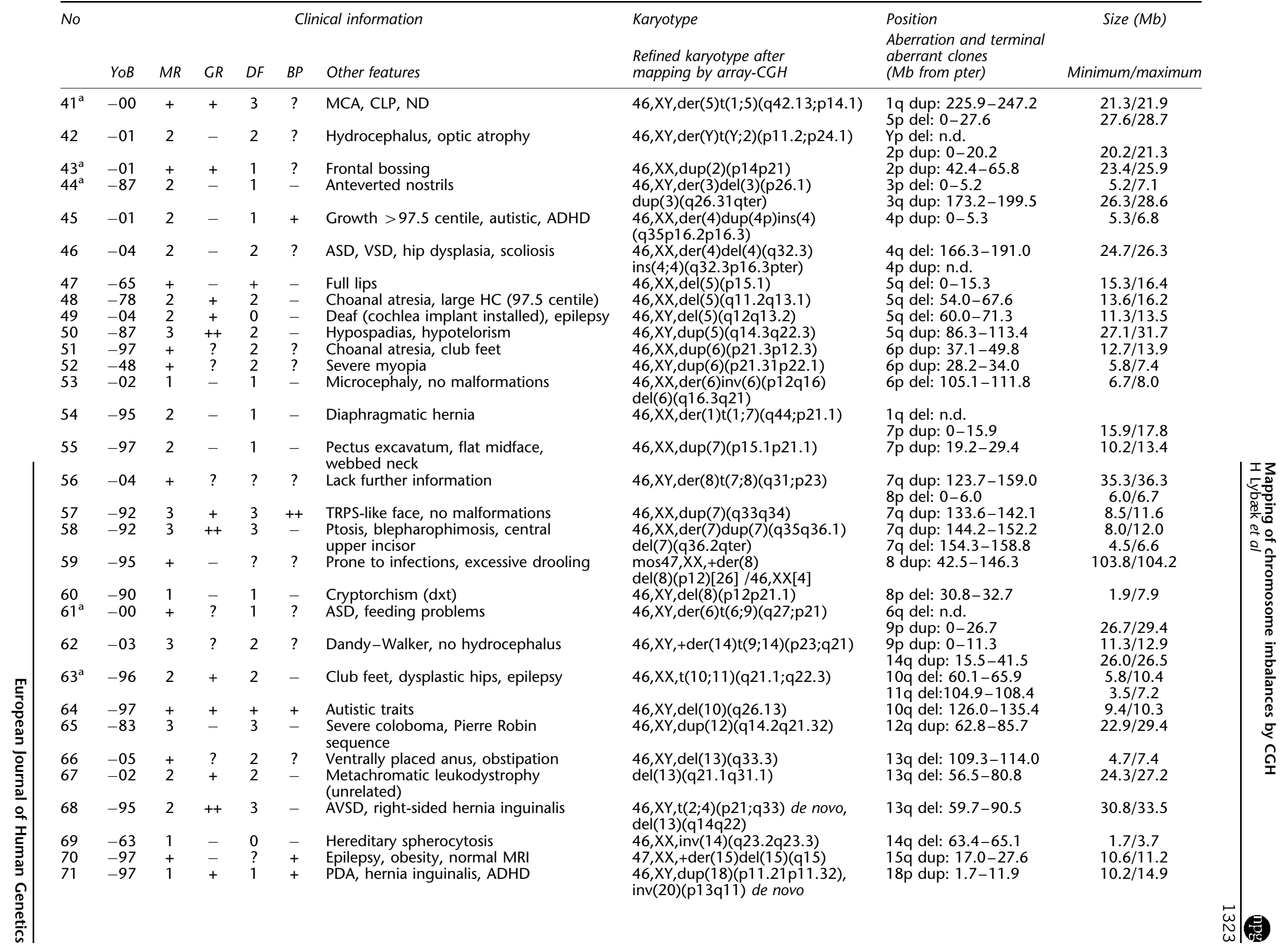


shown). Among the four patients, three imbalances were known to be de novo, whereas the aberration in case 80 was caused by a parental between-arm insertion. Even though the deletion on chromosome 14 in case 79 is close to the telomere, it would not have been detected by subtelomereFISH screening (Figure $3 b$ ). In case 77 , the finding of a deletion involving the $Z F H X 1 B$ gene was expected on clinical grounds because the patient had Mowat-Wilson syndrome, known to be caused by haploinsufficiency of this gene. ${ }^{21}$ This finding was also verified by a BAC-FISH probe against the ZFHX1B locus. None of the deletions were picked up by HR-CGH analysis, even at lower confidence intervals, but the duplication in case 80 was seen when the confidence intervals were set at $95 \%$. However, the amount of noise on the HR-CGH profiles at such low confidence intervals makes analysis at this level of sensitivity unfeasible.

\section{Discussion}

The main purpose of this study was fine mapping for improved genotype-phenotype information on a wellcharacterised group of patients with minor and cryptic chromosomal imbalances diagnosed during a 6-year period. In addition, the performance of the two CGH techniques could be compared.

In patients with normal karyotypes and a combination of mental retardation and dysmorphic signs and/or malformations, the detection rate of HR-CGH was $7.2 \%$. Our results are comparable to a group of 332 patients from five different European studies, where the $1 \mathrm{Mb}$ array-CGH diagnostic detection rate, all data combined, was $8.4 \%{ }^{10}$ This does not indicate that array-CGH is only slightly better in detecting clinically relevant genomic imbalances than HR-CGH. The yield of different diagnostic approaches is dependent on patient ascertainment, although the influence of patient selection on the detection rate might be smaller than previously thought. ${ }^{22}$ In the five European studies summarised above, the $1 \mathrm{Mb}$ array-CGH diagnostic detection rates varied from $8 \%{ }^{10}$ to $16 \% .{ }^{23}$ Of note, some of the patients in these studies had been pre-screened for subtelomeric imbalances, and elimination of these ten patients from our cohort would have reduced our diagnostic detection rate to $5.4 \%$. In addition, our results show that HR-CGH may overlook even visible subtelomeric imbalances. Therefore, HR-CGH should be combined with conventional G-banding whenever feasible. In a subgroup of 20 patients with normal findings on G-banding and HR-CGH but with a strong clinical suspicion of a genomic imbalance, four patients (20\%) had a genomic imbalance. The sample is too small to conclude if this high detection rate is a random finding or due to skilled patient selection. 


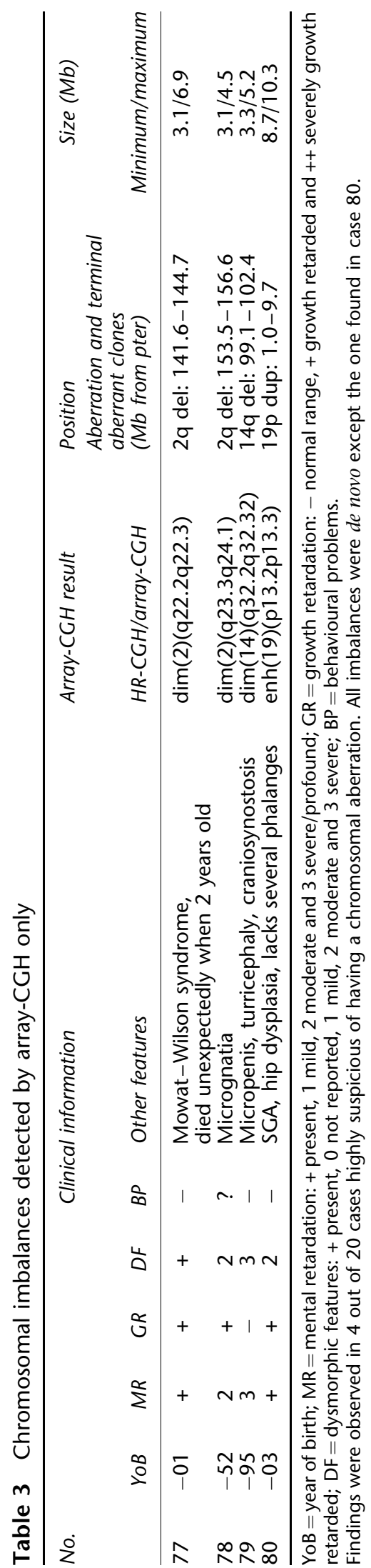

Among the 80 patients with genomic imbalances, findings were on all chromosomes, in agreement with other $1 \mathrm{Mb}$ array-CGH studies. ${ }^{10}$ The largest numbers of aberrations, with deletions being as common as duplications, were seen on chromosomes 7 and 8. On chromosome 19, our most gene-dense chromosome, only duplications were found. Another interesting observation was that minor but visible duplications were as common as deletions, whereas cryptic deletions were 10 times more common than cryptic duplications. One explanation may be that deletions are easier to detect than duplications because the actual change in DNA amount is 100\% for deletions and $50 \%$ for duplications. It is also possible that small duplications are less likely to cause a phenotype that makes the patient look 'chromosomal', that is, reduces the chance of ascertainment.

Although useful, it is difficult to evaluate the clinical significance of deletions and duplications based only on size, especially until we have a more extensive and indepth knowledge about copy number variations (CNVs) in the human genome. The number of microdeletion syndromes that are solely or largely caused by the absence of one gene among many is increasing (eg EHMT1 in 9q34 deletions, ${ }^{24}$ RAI1 in Smith-Magenis syndrome, ${ }^{25}$ and LIS1 in Miller-Dieker syndrome). Therefore, parental DNAs should be available to verify that a finding is de novo. Using different types of arrays, others have found that CNVs occur probably in all healthy individuals. ${ }^{26-28}$ It is not yet clear how much of the human genome that is quite tolerant to $\mathrm{CNV}$, but it appears to be at least $12 \% .^{28}$ Eventually, we will know the identity of most genes that cannot be duplicated or deleted without affecting normal development or brain function.

Looking at individual patients with interesting phenotypes, it is noted that in case 3 , an $\sim 9.3 \mathrm{Mb}$ deletion of 2q32.3-2q33.1 was associated with Duane anomaly, autistic traits and ataxia. This deletion on chromosome 2 is $\sim 5 \mathrm{Mb}$ distal to the published locus for Duane retraction syndrome type 2 (DURS2), mapped by linkage analysis to $2 \mathrm{q} 31 .^{29}$ Maybe the deletion is associated with an inversion disrupting the DURS2 gene. In case 4 with severe autism and self-destructive behaviour, a terminal deletion on $2 \mathrm{q}$ was found, a region previously found to be deleted in autistic patients. ${ }^{30}$ A patient with a similar-sized terminal $2 \mathrm{q}$ deletion who had severe behavioural disturbance was recently described. ${ }^{31}$ The deletion in case 17 , where bifid thumbs and bifid first toes were found in addition to epilepsy and a small penis, includes the GLI3 gene. Deletions or mutations in this gene are associated with Greig cephalopolysyndactyly (OMIM 175700) and PallisterHall syndromes (OMIM 146510), and epilepsy and a small penis are also features of the latter. The small deletion of $7 q 22.1-7 q 31.1$ in case 19 includes reelin $(R E L N)$, a gene that may be mutated in Norman-Roberts-type lissencephaly (OMIM 257320) and thought to be associated with autism 

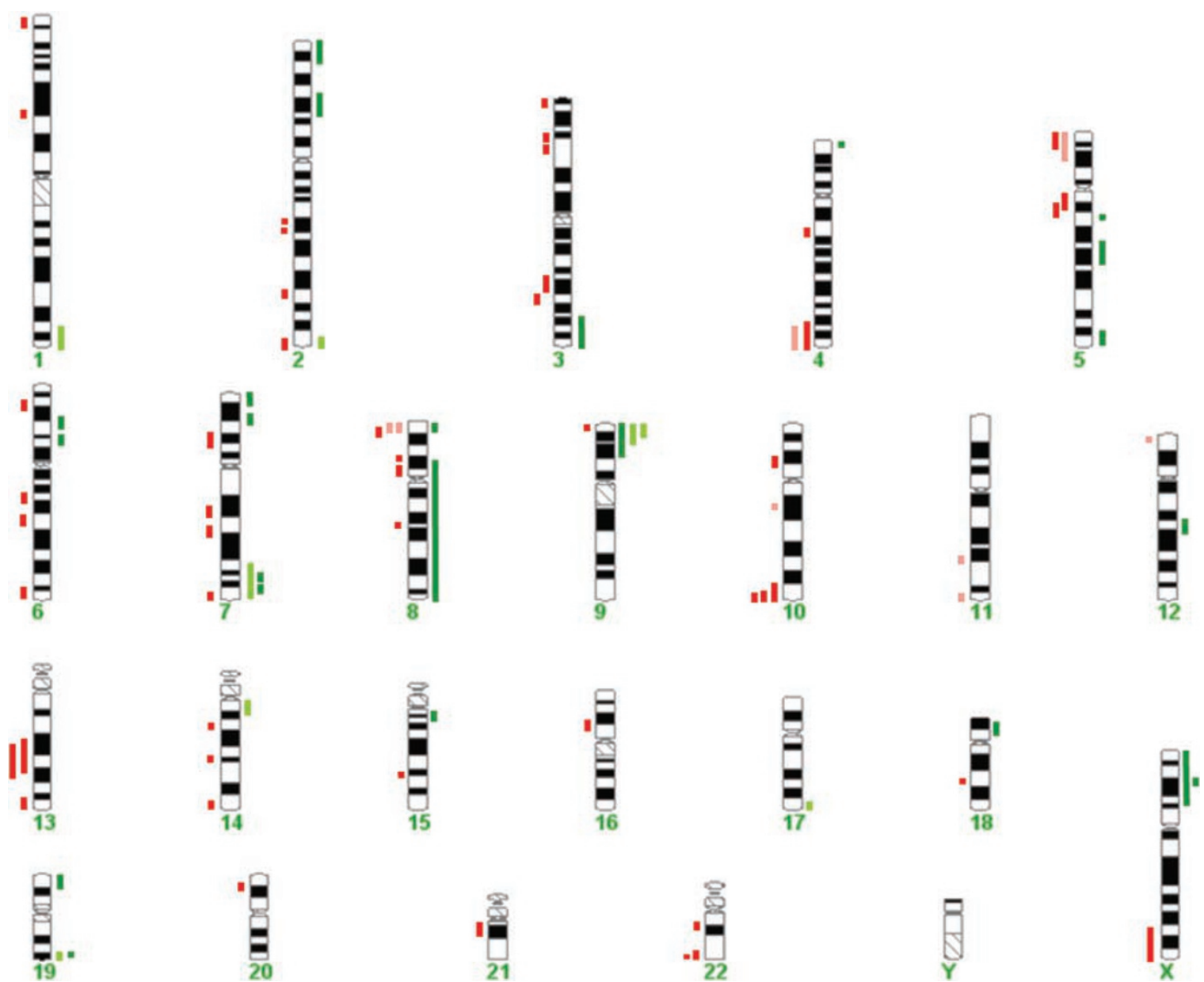

Figure 2 Overview of chromosomal imbalances mapped by $1 \mathrm{Mb}$ array-CGH: 400-band chromosome ideograms are shown with duplications drawn as green lines and deletions as red lines. Imbalances caused by translocations are shown in a lighter colour.

and schizophrenia. This patient is growth retarded (length just below the 2.5 centile) but has a head circumference in the upper normal range (75-90 centile). Anterior synechiae and microcoria are peculiar features. In case 48 , an $\sim 13.6 \mathrm{Mb}$ deletion of $5 \mathrm{q} 11.2-5 \mathrm{q} 13.1$ was associated with choanal atresia, macrocephaly and pronounced dysmorphism. In case 49 , a similar-sized $(\sim 11.3 \mathrm{Mb})$ but $\sim 6 \mathrm{Mb}$ more distal deletion (5q12-5q13.2) was not associated with dysmorphic features but congenital deafness. The deletions overlap with $\sim 8 \mathrm{Mb}$. No dominant deafness genes or genes associated with choanal atresia or macrocephaly are known to be located in the interval. One gene with high expression in the central nervous system (CNS) that is located in the 3$4 \mathrm{Mb}$ region deleted in case 49 but not in case 48 is MAP1B (microtubule-associated protein 1B). Heterozygosity for an insertional knockout in this gene leads to severe CNS affection in mice. ${ }^{32}$ Finally, case 33 (Table 1), with occult mosaicism for trisomy 14 , deserves to be mentioned, as this is a rare type of trisomy mosaicism..$^{33}$ It illustrates that such mosaicism, similar to our three patients with mosaic trisomy 9 (cases 25-27; Table 1), can be detected by CGH using blood sample DNA even though metaphase studies of blood lymphocyte cultures are completely normal. ${ }^{10,34}$ The lowest HR-CGH-detected mosaicism grade was $15 \%$, and this was found in a patient with trisomy 9 (case 27). Previously, mosaicism grade as low as $8 \%$ has been detected by $1 \mathrm{Mb}$ array-CGH analysis. ${ }^{10}$

This work adds to our knowledge on minor and cryptic chromosome imbalances that cause varying degrees of mental impairment, malformations and/or dysmorphism. Mapping of genomic imbalances having distinct phenotypes may be useful not only for evaluation of the clinical importance of a de novo imbalance, but also for narrowing down regions of particular interest when searching for genes whose dosage is critical for normal development. 


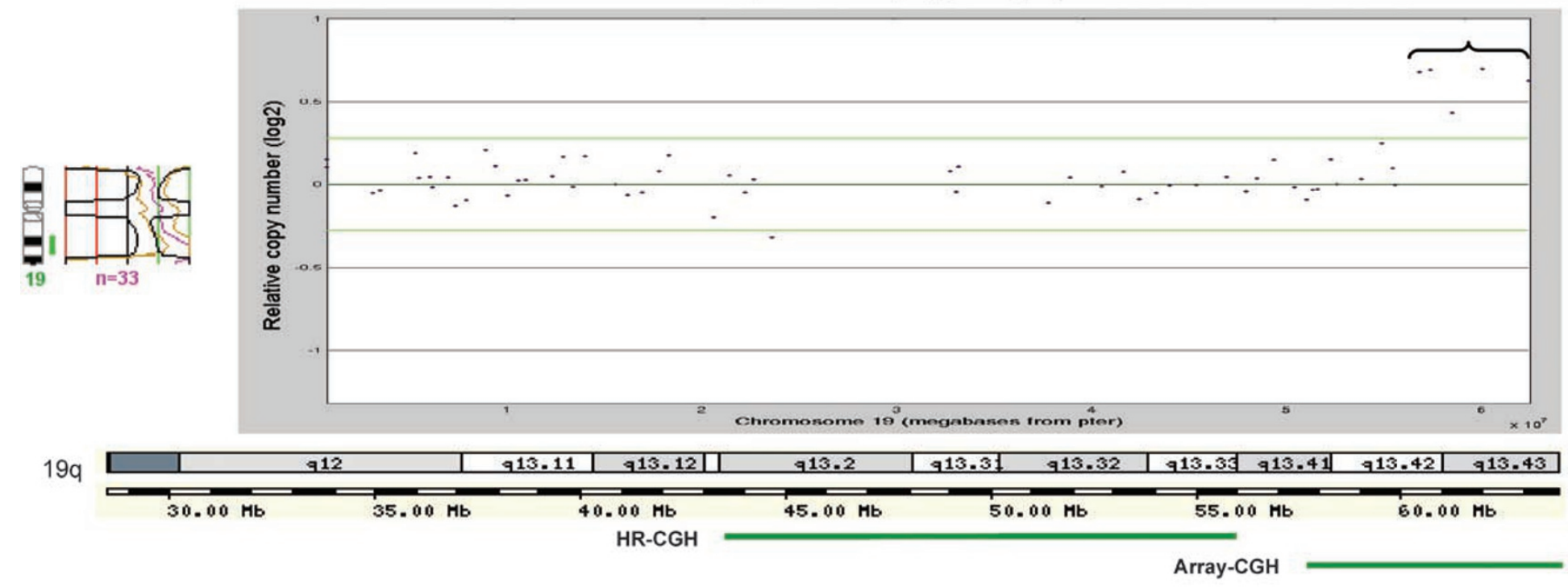

b

Case 79, .rev ish $\operatorname{dim}(14)(\mathrm{q} 32.2 \mathrm{q} 32.32)$

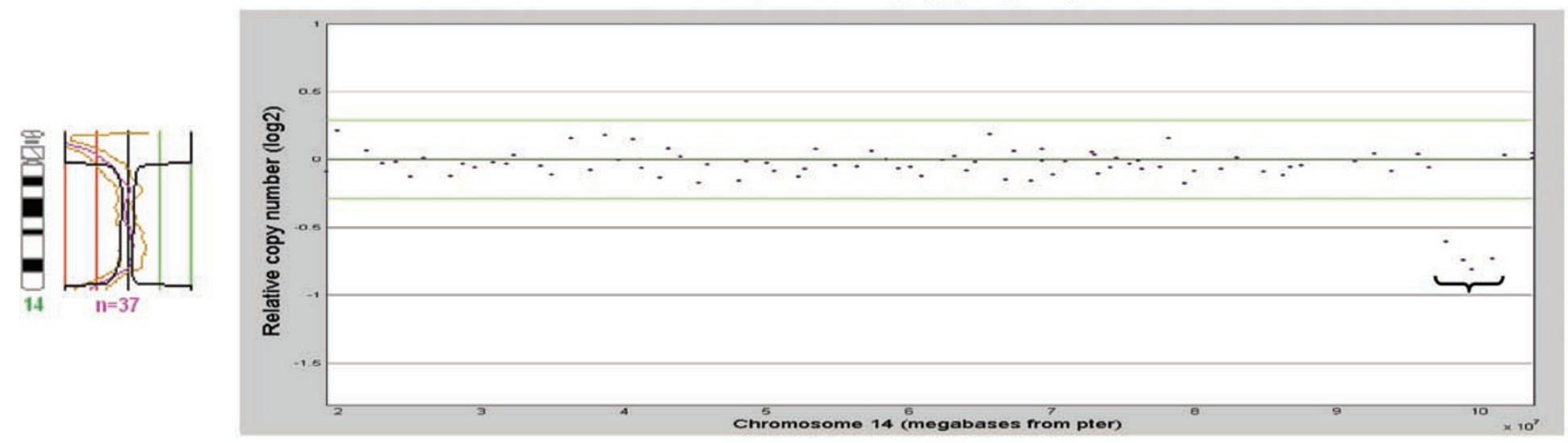

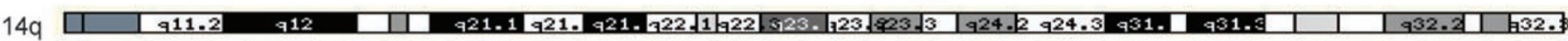

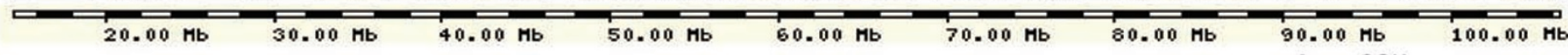

Figure 3 Inaccurate mapping of a chromosomal imbalance by HR-CGH (a) and array-CGH detection of a chromosomal imbalance in a case with normal findings on G-banding and HR-CGH (b): the $99.5 \%$ confidence interval HR-CGH ratio profiles together with the array-CGH profiles of case 72 (Table 2) and case 79 (Table 3 ) are shown. The imbalances mapped by $\mathrm{HR}-\mathrm{CGH}$ and array-CGH analyses are shown as green (gains) or red (losses) bars below an 850-band ideogram. (a) HR-CGH of case 72 indicated a non-terminal $19 \mathrm{q}$ duplication from $19 q 13.2$ to 19q13.3, whereas array-CGH showed a terminal duplication (dup(19)(q13.41qter)). (b) Normal findings by HR-CGH of case 79, whereas array-CGH detected a 14q deletion $(\operatorname{del}(14)(\mathrm{q} 32.2 \mathrm{q} 32.32))$ 


\section{Acknowledgements}

All the routine karyotyping was performed by the cytogenetic team working at the diagnostic laboratory at Haukeland University Hospital, led by Kjetil Solland. The genomic microarrays were produced by the Norwegian Microarray Consortium (NMC) supported by the functional genomics programme (FUGE) of the Research Council of Norway. We are especially grateful to the many clinicians who have contributed patients and phenotype information to this study: Marit Mork, Gro Zanussi, Inger Hellerdal-Rasmussen, Solfrid Larsen, Hallvard Reigstad, Christoffer Jonsrud, Gitte Vinther, Per Helge Kvistad, Nina Øyen, Finn Aspelund, Jarl Moldestad, Petter Strømme, Kristian Sommerfelt, Arve Vøllo, Arne Stubdal, Karen Helene Ørstavik, Gunnar Helland, Cathrine Helleland, Arvid Heidberg, Einar Heiervang, Andreas R Zagon, Trine Prescott, Trond Ludvigsen, Ineke Hogenesch, Lise Reindal, Carsten Muller, Gyri Aasland Gradek, Gyro Aas Herder, Arne Bjørnetun, Askild Stormorken, Henning S Aabech, Bente Ødegård, Viggo Lütschereth, Liv Marie Loegreid, Olav Roti, Tore Hågård, Martine Jaatun, Jan Olme, Bjug Åkre, Einar Bryne, Anne Lise Høyland, Kathinka Aslaksen, Asbjørg StrayPedersen, Sigurd Børsting, Ivar Moehle, Ewa Czarnecka, Kathrine Bjørgo, Kristoffer Helland-Hansen, Anne-Lene Søhoel, Valeria Marton, Peter Juliusson, Pål R Njølstad, Olav Skogen, and Irene Øversveen.

\section{References}

1 Gustavson KH, Holmgren G, Blomquist HK: Chromosomal aberrations in mildly mentally retarded children in a northern Swedish county. Upsala J Med Sci 1987; 44: 165-168.

2 Stromme P, Valvatne K: Mental retardation in Norway: prevalence and sub-classification in a cohort of 30037 children born between 1980 and 1985. Acta paediatr (Oslo, Norway) 1998; 87: 291-296.

3 Roeleveld N, Zielhuis GA, Gabreels F: The prevalence of mental retardation: a critical review of recent literature. Dev Med Child Neurol 1997; 39: 125-132.

4 van Karnebeek CD, Jansweijer MC, Leenders AG, Offringa M, Hennekam RC: Diagnostic investigations in individuals with mental retardation: a systematic literature review of their usefulness. Eur J Hum Genet 2005; 13: 6-25.

5 Rauch A, Hoyer J, Guth S et al: Diagnostic yield of various genetic approaches in patients with unexplained developmental delay or mental retardation. Am J Med Genet 2006; 140: 2063-2074.

6 Kirchhoff M, Rose H, Lundsteen C: High resolution comparative genomic hybridisation in clinical cytogenetics. J Med Genet 2001; 38: 740-744.

7 Ness GO, Lybaek H, Houge G: Usefulness of high-resolution comparative genomic hybridization $(\mathrm{CGH})$ for detecting and characterizing constitutional chromosome abnormalities. Am J Med Genet 2002; 113: 125-136.

8 Kirchhoff M, Gerdes T, Maahr J et al: Deletions below 10 megabasepairs are detected in comparative genomic hybridization by standard reference intervals. Genes Chromosomes Cancer 1999; 25: 410-413.

9 de Vries BB, Pfundt R, Leisink $M$ et al: Diagnostic genome profiling in mental retardation. Am J Hum Genet 2005; 77: 606-616.

10 Menten B, Maas N, Thienpont B et al: Emerging patterns of cryptic chromosomal imbalance in patients with idiopathic mental retardation and multiple congenital anomalies: a new series of 140 patients and review of published reports. J Med Genet 2006; 43: 625-633.

11 Schoumans J, Ruivenkamp C, Holmberg E, Kyllerman M, Anderlid BM, Nordenskjold M: Detection of chromosomal imbalances in children with idiopathic mental retardation by array based comparative genomic hybridisation (array-CGH). J Med Genet 2005; 42: 699-705.

12 Shaffer LG, Bejjani BA: Medical applications of array CGH and the transformation of clinical cytogenetics. Cytogenet Genome Res 2006; 115: 303-309.

13 Shaw-Smith C, Redon R, Rickman L et al: Microarray based comparative genomic hybridisation (array-CGH) detects submicroscopic chromosomal deletions and duplications in patients with learning disability/mental retardation and dysmorphic features. J Med Genet 2004; 41: 241-248.

14 Vissers LE, de Vries BB, Osoegawa K et al: Array-based comparative genomic hybridization for the genomewide detection of submicroscopic chromosomal abnormalities. Am J Hum Genet 2003; 73: $1261-1270$

15 Shaffer LG, Kashork CD, Saleki R et al: Targeted genomic microarray analysis for identification of chromosome abnormalities in 1500 consecutive clinical cases. J Pediatr 2006; 149: 98-102.

16 Kirchhoff M, Gerdes T, Rose H, Maahr J, Ottesen AM, Lundsteen $\mathrm{C}$ : Detection of chromosomal gains and losses in comparative genomic hybridization analysis based on standard reference intervals. Cytometry 1998; 31: 163-173.

17 Fiegler H, Carr P, Douglas EJ et al: DNA microarrays for comparative genomic hybridization based on DOP-PCR amplification of BAC and PAC clones. Genes Chromosomes Cancer 2003; 36: 361-374.

18 Meza-Zepeda LA, Kresse SH, Barragan-Polania AH et al: Array comparative genomic hybridization reveals distinct DNA copy number differences between gastrointestinal stromal tumors and leiomyosarcomas. Cancer Res 2006; 66: 8984-8993.

19 Wang J, Meza-Zepeda LA, Kresse SH, Myklebost O: M-CGH: analysing microarray-based CGH experiments. BMC Bioinform 2004; 5: 74.

20 Ballif BC, Wakui K, Gajecka M, Shaffer LG: Translocation breakpoint mapping and sequence analysis in three monosomy $1 \mathrm{p} 36$ subjects with $\operatorname{der}(1) \mathrm{t}(1 ; 1)(\mathrm{p} 36 ; \mathrm{q} 44)$ suggest mechanisms for telomere capture in stabilizing de novo terminal rearrangements. Hum Genet 2004; 114: 198-206.

21 Zweier C, Albrecht B, Mitulla B et al: 'Mowat-Wilson' syndrome with and without Hirschsprung disease is a distinct, recognizable multiple congenital anomalies-mental retardation syndrome caused by mutations in the zinc finger homeo box 1B gene. Am J Med Genet 2002; 108: 177-181.

22 Hoyer J, Dreweke A, Becker C et al: Molecular karyotyping in patients with mental retardation using $100 \mathrm{~K}$ single-nucleotide polymorphism arrays. J Med Genet 2007; 44: 629-636.

23 Rosenberg C, Knijnenburg J, Bakker E et al: Array-CGH detection of micro rearrangements in mentally retarded individuals: clinical significance of imbalances present both in affected children and normal parents. J Med Genet 2006; 43: 180-186.

24 Kleefstra T, Brunner HG, Amiel J et al: Loss-of-function mutations in euchromatin histone methyl transferase 1 (EHMT1) cause the 9q34 subtelomeric deletion syndrome. Am J Hum Genet 2006; 79: 370-377.

25 Slager RE, Newton TL, Vlangos CN, Finucane B, Elsea SH: Mutations in RAI1 associated with Smith-Magenis syndrome. Nat Genet 2003; 33: 466-468.

26 Iafrate AJ, Feuk L, Rivera MN et al: Detection of large-scale variation in the human genome. Nat Genet 2004; 36: 949-951.

27 Sebat J, Lakshmi B, Troge J et al: Large-scale copy number polymorphism in the human genome. Science 2004; 305: 525-528.

28 Redon R, Ishikawa S, Fitch KR et al: Global variation in copy number in the human genome. Nature 2006; 444: 444-454.

29 Appukuttan B, Gillanders E, Juo SH et al: Localization of a gene for Duane retraction syndrome to chromosome 2q31. Am J Hum Genet 1999; 65: 1639-1646.

30 Jacquemont $\mathrm{ML}$, Sanlaville $\mathrm{D}$, Redon $\mathrm{R}$ et al: Array-based comparative genomic hybridisation identifies high frequency of cryptic chromosomal rearrangements in patients with syndromic autism spectrum disorders. J Med Genet 2006; 43: 843-849.

31 Kitsiou-Tzeli S, Sismani C, Ioannides M et al: Array-CGH analysis and clinical description of $2 \mathrm{q} 37.3$ de novo subtelomeric deletion. Eur J Med Genet 2007; 50: 73-78.

32 Edelmann W, Zervas M, Costello P et al: Neuronal abnormalities in microtubule-associated protein 1B mutant mice. Proc Natl Acad Sci USA 1996; 93: 1270-1275.

33 Lynch MF, Fernandes CJ, Shaffer LG, Potocki L: Trisomy 14 mosaicism: a case report and review of the literature. J Perinatol 2004; 24: 121-123.

34 Ballif BC, Rorem EA, Sundin K et al: Detection of low-level mosaicism by array CGH in routine diagnostic specimens. Am J Med Genet A 2006; 140: 2757-2767. 